\title{
Effects of person-centered care on residents and staff in aged-care facilities: a systematic review
}

This article was published in the following Dove Press journal:

Clinical Interventions in Aging

27 December 2012

Number of times this article has been viewed

\section{Sonya Brownie \\ Susan Nancarrow}

School of Health and Human Sciences, Southern Cross University, Lismore, NSW, Australia
Correspondence: Sonya Brownie School of Health and Human Sciences, Southern Cross University, PO Box I57, Lismore, NSW, 2480, Australia

Tel +6I2 66203948

Fax +6I 266203307

Email sonya.brownie@scu.edu.au
Background: Several residential aged-care facilities have replaced the institutional model of care to one that accepts person-centered care as the guiding standard of practice. This culture change is impacting the provision of aged-care services around the world. This systematic review evaluates the evidence for an impact of person-centered interventions on aged-care residents and nursing staff.

Methods: We searched Medline, Cinahl, Academic Search Premier, Scopus, Proquest, and Expanded Academic ASAP databases for studies published between January 1995 and October 2012, using subject headings and free-text search terms (in UK and US English spelling) including person-centered care, patient-centered care, resident-oriented care, Eden Alternative, Green House model, Wellspring model, long-term care, and nursing homes.

Results: The search identified 323 potentially relevant articles. Once duplicates were removed, 146 were screened for inclusion in this review; 21 were assessed for methodological quality, resulting in nine articles (seven studies) that met our inclusion criteria. There was only one randomized, controlled trial. The majority of studies were quasi-experimental pre-post test designs, with a control group $(n=4)$. The studies in this review incorporated a range of different outcome measures (ie, dependent variables) to evaluate the impact of person-centered interventions on aged-care residents and staff. One person-centered intervention, ie, the Eden Alternative, was associated with significant improvements in residents' levels of boredom and helplessness. In contrast, facility-specific person-centered interventions were found to impact nurses' sense of job satisfaction and their capacity to meet the individual needs of residents in a positive way. Two studies found that person-centered care was actually associated with an increased risk of falls. The findings from this review need to be interpreted cautiously due to limitations in study designs and the potential for confounding bias.

Conclusion: Typically, person-centered interventions are multifactorial, comprising: elements of environmental enhancement; opportunities for social stimulation and interaction; leadership and management changes; staffing models focused on staff empowerment; and assigning residents to the same care staff and an individualized philosophy of care. The complexity of the interventions and range of outcomes examined makes it difficult to form accurate conclusions about the impact of person-centered care interventions adopted and implemented in aged-care facilities. The few negative consequences of the introduction of person-centered care models suggest that the introduction of person-centered care is not always incorporated within a wider "hierarchy of needs" structure, where safety and physiological need are met before moving onto higher level needs. Further research is necessary to establish the effectiveness of these elements of person-centered care, either singly or in combination.

Keywords: individualized care, nursing homes, culture change in care homes, residential aged-care facilities 


\section{Introduction}

Residential aged-care facilities have been viewed as places of long-term treatment and therapy dominated by the biomedical model that values efficiency, consistency, and hierarchical decision-making. ${ }^{1}$ Older people (particularly baby-boomers) have expressed a strong preference for alternative forms of aged care and accommodation, and a greater ability to exercise control over where they live and the nature and quality of services they will receive. ${ }^{2,3}$ The aged-care sector is under pressure to provide a range of innovative and contemporary models of care that enhance emotional, physical, and functional well-being through enriched, integrative environments. ${ }^{4}$ Residential aged-care facilities are adopting a new paradigm for long-term care that is part of a culture change that accepts person-centered care as the guiding or defining standard of practice.

Carl Rogers, one of the 20th century's most influential humanistic psychologists, founded the "person-centered" approach to care. Rogers proposed that a person-centered approach, based on acceptance, caring, empathy, sensitivity, and active listening, promotes optimal human growth. He believed that in order to actualize human growth in late life, individuals should have access to, and opportunities for, ongoing learning, personal challenges, and close and intimate relationships. ${ }^{5}$ Furthermore, he argued that human capacity for growth does not diminish with age, nor does the need for growth become less relevant as we age. The emphasis of person-centered care is on the well-being and quality of life as defined by the individual. ${ }^{6}$

In 1991, Dr William Thomas, a US geriatrician, pioneered the Eden Alternative, which is a person-centered approach to long-term residential aged care. His central premise was that "every creature has a habitat in which it thrives, and one in which it withers. Human beings wither in institutions". ${ }^{7}$ Like Rogers, Thomas believed that late life was an active phase of the aging trajectory, and to that end he wanted to create a human habitat that would optimize growth for long-term care residents. His aim was to deinstitutionalize aged-care facilities by enlivening the environment with children, animals, and plants to create an atmosphere reminiscent of home. The Eden Alternative is based on a set of ten principles that provides aged-care facilities with a blueprint for organizational reform designed to create a warm and caring "home" that enriches the lives of all who live and work in it. The principles identify the challenges and the solutions to providing a person-centered care environment for aged-care residents.

Since the inception of the Eden Alternative more than three decades ago, variations of person-centered models of aged care have been developed and implemented in aged-care facilities around the world, but predominantly in the US. The Wellspring and Green House models are examples of two models of culture change with person-centered care at the core of their philosophy. Wellspring (the Wellspring Innovative Solutions Inc) is a confederation of 11 freestanding not-for-profit homes in Wisconsin that was founded in 1994 and became fully operational in 1998. It seeks to "change the clinical quality of care and the organizational culture in its member facilities". The Green House model is a small-scale, purpose-built, aged-care facility that accommodates up to ten residents with high-care nursing needs. Dr Thomas developed this concept to harmonize the philosophy of the Eden Alternative with the built environment, in an attempt to replicate a home-like setting where all meals are prepared on site, residents have access to outdoor spaces, and nurses have broad roles, which include meal preparation and management of medication, in addition to meeting the care needs of the residents.

A study conducted in 2002 in North Carolina ${ }^{9}$ found that there was widespread interest specifically in environmental enhancement interventions in aged-care facilities. The majority of facilities (71\%) that participated in the study reported that they were planning or implementing some sort of environmental enhancement, eg, introduction of plants and animals. More recently, the 2007 National Survey of Nursing Homes examined the extent to which nursing homes in the US had adopted principles of culture change and were practicing resident-centered care. ${ }^{10} \mathrm{Of}$ the 1435 nursing homes that were surveyed, $5 \%$ had "completely embraced culture change", $25 \%$ were "very committed to adopting culture change", $25 \%$ had "made few changes but management were committed to adopting culture change", and the majority (43\%) of nursing homes surveyed stated that their facility "had not adopted any aspect of culture change or very little, and that management were not very committed, if at all, to culture change practices".

Despite the emergence of person-centered approaches and philosophies of care in residential aged-care facilities, there is a paucity of data on the impact of these approaches on the well-being of residents and staff. The purpose of this review was to evaluate the impact of person-centered care approaches on residents and staff in residential aged-care facilities.

\section{Literature search strategy and selection criteria}

The search strategy, including a list of the databases and search terms used for this review along with the number of articles found using this method is shown in Table 1. This method yielded 323 full-text articles. Once duplicates 
Table I Databases and search terms used to identify literature for review

\begin{tabular}{|c|c|c|}
\hline Database & Search terms & $\begin{array}{l}\text { Citations } \\
\text { reviewed }\end{array}$ \\
\hline Medline & $\begin{array}{l}\text { Patient-centered care [MeSH] OR "person-cent\#red care" OR "patient-focus\#ed care" OR "resident- } \\
\text { cent\#red care" OR "relationship-cent\#red care" OR "individuali\#ed care" OR "resident-oriented care" }\end{array}$ & 61 \\
\hline \multirow[t]{2}{*}{ AND } & Homes for the aged $[\mathrm{MesH}]$ OR nursing homes $[\mathrm{MesH}]$ OR residential facilities $[\mathrm{MesH}] \mathrm{OR}$ & \\
\hline & long-term care $[\mathrm{Mes} \mathrm{H}] \mathrm{OR}$ assisted living facilities $[\mathrm{MesH}]$ & \\
\hline OR & "Eden Alternative" OR "Green House model" OR "Wellspring model" & \\
\hline Limiters & 1995-2012; full text, English & \\
\hline Cinahl & $\begin{array}{l}\text { Patient-centered care [Heading] OR "person-cent\#red care" OR "patient-focus\#ed care" OR "resident- } \\
\text { cent\#red care" OR "relationship-cent\#red care" OR "individuali\#ed care" OR "resident-oriented care" }\end{array}$ & 37 \\
\hline \multirow[t]{2}{*}{ AND } & Nursing homes [Heading] OR residential care [Heading] OR residential facilities [Heading] & \\
\hline & OR housing for the elderly [Heading] OR "long-term care" & \\
\hline OR & "Eden Alternative" OR "Green House Model" OR "Wellspring Model” & \\
\hline Limiters & 1995-2012; full text, English, research article [Publication type] & \\
\hline $\begin{array}{l}\text { Academic search } \\
\text { premier }\end{array}$ & $\begin{array}{l}\text { Patient-centered care [Subject term] OR "person-cent\#red care" OR "patient-focus\#ed care" OR "resident- } \\
\text { cent\#red care" OR "relationship-cent\#red care" OR "individuali\#ed care" OR "resident-oriented care" }\end{array}$ & 79 \\
\hline AND & $\begin{array}{l}\text { Residential care [Subject term] OR nursing care facilities [Subject term] OR "long-term care facilities" } \\
\text { [Subject term] OR old age homes [Subject term] OR nursing home* OR "residential care facilities" }\end{array}$ & \\
\hline OR & "Eden Alternative" OR "Green House model" OR "Wellspring model" & \\
\hline Limiters & 1995-2012; full text, English, scholarly (peer-reviewed) journals & \\
\hline \multirow[t]{2}{*}{ Scopus } & "Person-cent?red care" OR "patient-focus?ed care" OR "resident-cent?red care" OR & 31 \\
\hline & "relationship-cent?red care" OR “individuali?ed care” OR "resident-oriented care" & \\
\hline \multirow[t]{2}{*}{ AND } & Nursing home* OR "residential care" OR "residential facilities" OR "housing for the elderly" & \\
\hline & OR "long-term care" OR "long-term care facilities" & \\
\hline OR & "Eden Alternative" OR "Green House model" OR "Wellspring model" & \\
\hline Limiters & 1995-2012, Article [Publication type], article title, abstract and keywords & \\
\hline \multirow[t]{3}{*}{ ProQuest } & Person-centred care [Search term] OR patient-centred care [Search term] OR "person-centered care" & 38 \\
\hline & OR "patient-centered care" OR "patient-focus?ed care" OR "resident-cent?red care" & \\
\hline & OR "relationship-cent?red care" OR "individuali?ed care" OR "resident-oriented care" & \\
\hline \multirow[t]{2}{*}{ AND } & Residential aged care [Search term] OR aged care homes [Search term] OR homes for the aged & \\
\hline & [Search term] OR nursing homes for the elderly [Search term] OR nursing home* [Search term] & \\
\hline OR & "Eden Alternative" OR "Green House model" OR "Wellspring model" & \\
\hline Limiters & 1995-2012, full text, English, peer reviewed, article [Document type] & \\
\hline Expanded academic & Personalised care [Keyword] OR patient centred care [Keyword] OR patient centeredness [Keyword] & 77 \\
\hline \multirow[t]{2}{*}{ ASAP } & OR patient centered approach [Keyword] OR individuali!ed care [Keyword] OR patient focus!ed care & \\
\hline & [Keyword] OR "resident-oriented care" OR "relationship-cent!red care" OR person-cent!red care & \\
\hline \multirow[t]{2}{*}{ AND } & "Aged care residents" [Keyword] OR aged care facilities [Keyword] OR nursing home* [Keyword] OR & \\
\hline & "nursing home residents" [Keyword] OR residential care [Keyword] OR long term care facilities [Keyword] & \\
\hline OR & "Eden Alternative" OR "Green House model" OR "Wellspring model” & \\
\hline Limiters & 1995-2012, article [Document type], full text, peer-reviewed & \\
\hline \multicolumn{2}{|c|}{ Total records identified after database searching } & 323 \\
\hline \multicolumn{2}{|c|}{ Total records after duplicates removed } & 146 \\
\hline
\end{tabular}

( $\mathrm{n}=177$ articles) were removed, 146 articles were screened for inclusion. The majority of the articles $(n=125)$ were secondary sources of literature or studies focused on hospitals and palliative care, and on secondary issues related to culture change in residential aged care, eg, families' perceptions about the impact of culture change interventions and the process of reforming care models in aged-care facilities. The remaining 21 articles were screened for inclusion in this review.

The inclusion criteria were:

- Study designs, ie, experimental design studies, including pre-post test design studies with or without a control group or randomized trials.
- Intervention, ie, person-centered approaches to residential aged care, including interventions focused on enhancing residents' autonomy, choice, sense of personal control, independence and interactions with residents and staff. Key phrases in studies that reflect the objectives of these interventions included person-centered care, patient-centered care, quality of life, quality of health care, individuality in old age, satisfaction with care, and organizational culture.

- Subjects and setting, ie, residents in a long-term aged-care facility (nursing home) and/or nursing staff. 
- Publication type, ie, peer-reviewed articles in scholarly journals published in English.

Both authors independently read each of the 21 full-text studies and applied the inclusion criteria to identify experimental studies that were eligible for inclusion in the review. Where there was a discrepancy of views related to the eligibility of studies for inclusion, both authors discussed and justified their decision-making until they reached agreement about whether to include or exclude an article.

Twelve articles were excluded for the following reasons:

- Study designs and/or interventions inconsistent with inclusion criteria ${ }^{4,6,11-19}$

- Subjects and setting inconsistent with inclusion criteria $^{20}$

The reference lists of the nine articles that met the inclusion criteria for this review were searched for additional relevant articles that might have been missed using the literature search strategy method. Articles with a combination of the search terms (used in the initial literature search, as shown in Table 1) in the article title were retrieved and screened using the inclusion criteria. This process identified three ${ }^{21-23}$ potentially relevant articles, but none were experimental studies. See Figure 1 for a modified Preferred Reporting Items for Systematic Reviews and Meta-Analyses (PRISMA) flow diagram.

\section{Results}

Nine articles ( $\mathrm{n}=7$ studies) met the inclusion criteria for this review. None of the existing literature on the Wellspring

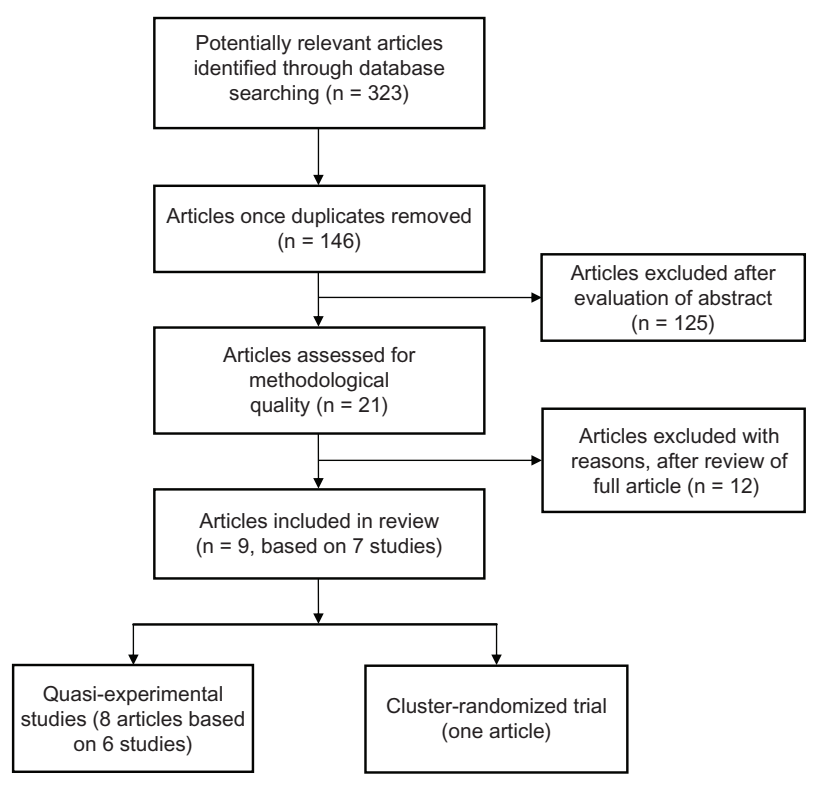

Figure I Modified Preferred Reporting Items for Systematic Reviews and Meta-Analyses (PRISMA) flow diagram of article screening and selection. model met the inclusion criteria. One study used the term "resident-oriented care", which was consistent with the principles of person-centered care. Central to person-centered interventions is a commitment by the aged-care facility to adopt and implement personalized care intended to enhance residents' autonomy, choice, sense of personal control, independence, and interactions with residents and staff. Residential aged-care facilities were commonly referred to as nursing homes or long-term care facilities in the literature.

\section{Appraisal of study design quality}

The methodological quality of the studies was appraised using the "appraisal of randomized and quasi-randomized controlled trials" developed by the Joanna Briggs Institute. ${ }^{24}$ This tool was deemed the most appropriate for the study designs included in this review (see Table 2). The majority of studies $(n=6)$ were quasi-experimental research designs; four were pre-post test studies with controls, ${ }^{25-30}$ and two were prepost test studies without a control group. ${ }^{31,32}$ In general, these studies incorporated quantitative data collection methods (mostly self-administered questionnaires and Minimum Data Set (MDS) data) with qualitative data collection methods (focus groups and interviews). ${ }^{26,30,32}$ The remaining study was a cluster-randomized, controlled trial designed to evaluate the impact of a person-centered intervention on dementia-related behavior, ${ }^{33}$ such as agitation, and other secondary outcomes, including quality of life and incidence of falls. In three of the studies, the control and intervention groups varied at baseline for age, ethnicity, and/or dependency status. ${ }^{28-30} \mathrm{It}$ was difficult to determine consistency and comparability of the interventions in several of the cases, and these differences were poorly explored in most of the studies.

\section{Data extraction}

The heterogeneity of study designs and the limited numeric outcome data prevented the statistical pooling of findings, so the findings are presented in narrative form, ie, in a table. The key study design features and results of these nine articles are presented in Table 3. One study conducted in The Netherlands yielded three articles, two ${ }^{25,26}$ of which report the same findings in relation to the impact of resident-oriented care on job characteristics of nursing staff, and the other ${ }^{27}$ reports the impact of this approach to care on residents' perception of quality of care, well-being, and their satisfaction with care. In Table 3, the two articles with the duplication of findings are presented together in one row, whereas the other findings ${ }^{27}$ (ie, the article by Boumans et $\mathrm{al}^{27}$ ) are presented in a separate row. This explains why the nine articles (based on seven studies) are reported in eight rows. 


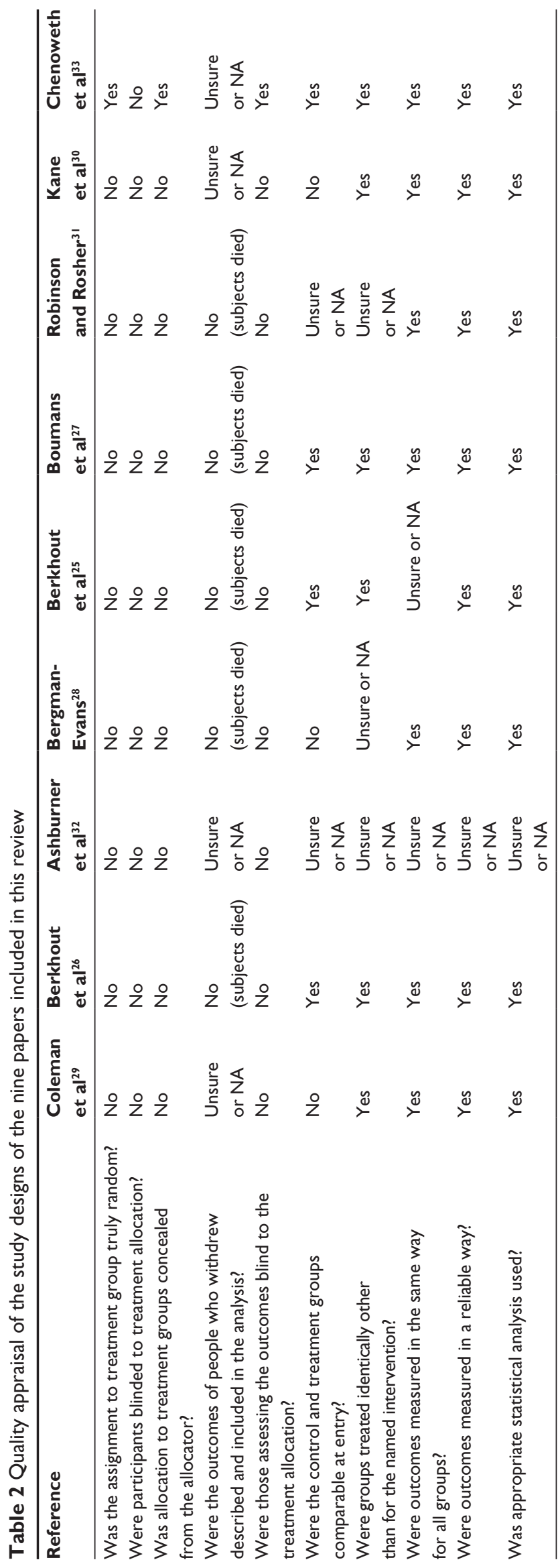

\section{Intervention characteristics}

Our literature review found that person-centered culture change interventions are not homogeneous or single-element interventions. Instead, they incorporate several features including:

- environmental enhancement (eg, plants and animals)

- opportunities for social stimulation and fulfilling relationships (visits by children and increased interaction with other residents and staff)

- continuity of resident care by assigning residents to the same care staff

- changes in management and leadership approaches (often devolved), with the introduction of democratized approaches to decision-making that involve residents and staff

- changes to staffing models focused on staff empowerment

- individualized (rather than institutionalized) humanistic philosophy of care.

The Eden Alternative is the only intervention identified in this review that articulates a framework (incorporating all these features) for a person-centered approach to caring for aged-care residents, and improving staff working conditions. In contrast, other types of person-centered interventions were facility-specific that focused on one or two features, ie, changes in management and leadership or continuity of resident care. The organizational framework used to implement person-centered interventions and the impact of these interventions on residents and staff is discussed in this section.

\section{Eden Alternative}

Three studies (conducted in the US) met the inclusion criteria for this review. ${ }^{28,29,31}$ Two studies reported improvements in residents' psychological well-being as measured by the prevalence of feelings of boredom, loneliness, helplessness, and depression in Eden Alternative facilities. ${ }^{28,31}$ These studies found statistically significant reductions in these feelings (except feelings of loneliness) for residents in Eden Alternative facilities when using validated psychological assessment tools, eg, the Geriatric Depression Scale, UCLA Loneliness Scale, and Cornell Depression Scale.

Coleman et $\mathrm{al}^{29}$ found that environmental enhancement was actually associated with adverse outcomes for residents in an Eden Alternative facility, compared with residents in a traditional (control) nursing home. This study found that residents in the Eden Alternative facility had a higher rate of falls ( $31 \%$ compared with $17 \%$ within a 30 -day period) 


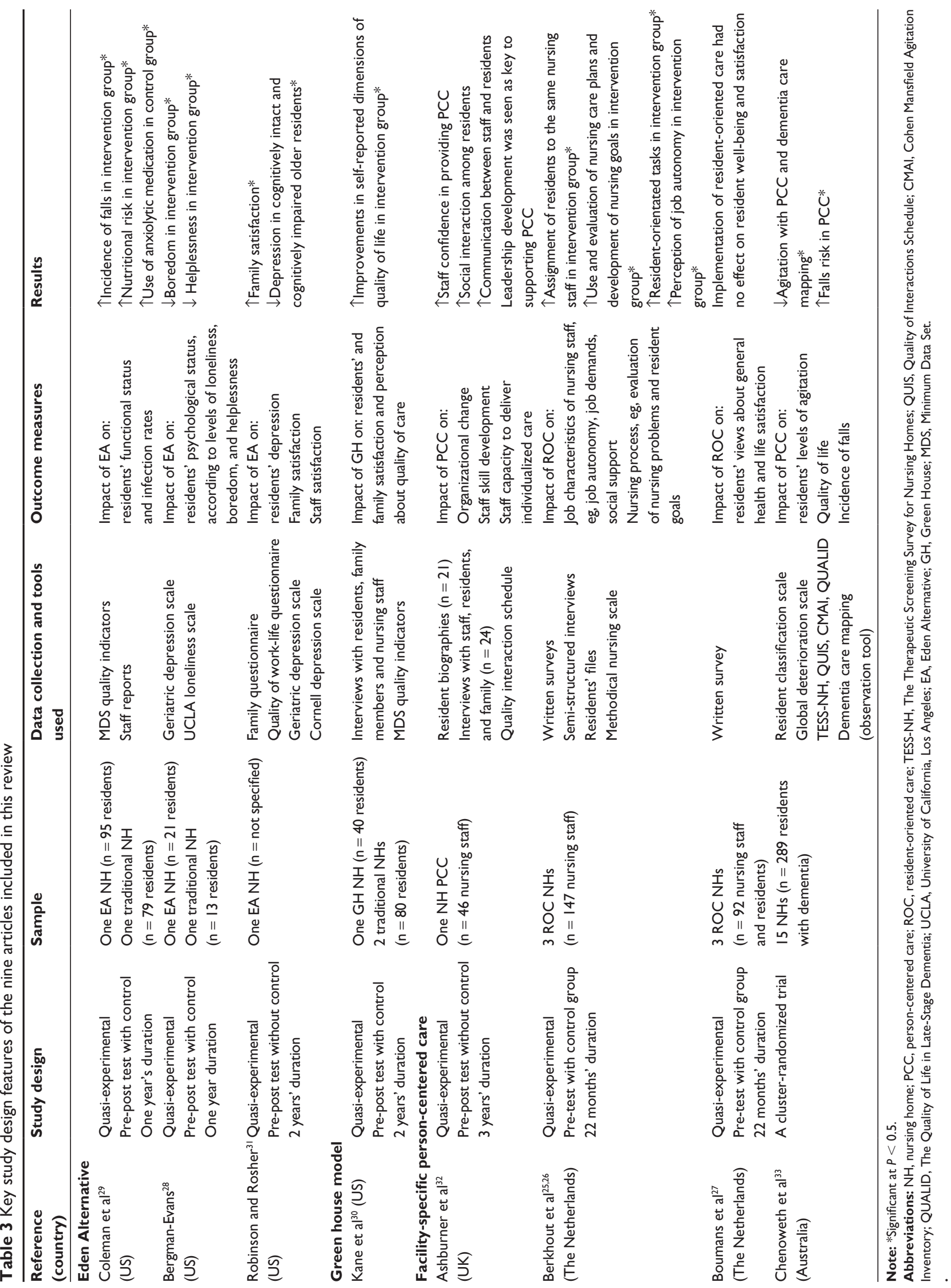


compared with controls. In this study, the residents in the Eden Alternative facility were on average younger than those in the control facility ( 82.6 years of age versus 88 years of age), with fewer impediments in relation to functional status (according to scores on an activity of daily living scale).

\section{Green House model}

One study (conducted in the US) met the inclusion criteria for this review. ${ }^{30}$ This two-year study compared residents in four 10-bed Green House homes $(\mathrm{n}=40)$ with two comparison sites; one was a nursing home $(n=40)$ located at the same facility as the Green House facility and the other facility $(\mathrm{n}=40)$ was more than $100 \mathrm{~km}$ away. All three facilities were operated by the same nonprofit owner in the US. Data were collected from interviews with residents, family, frontline nursing staff, and nursing home records (ie, MDS quality indicators) at baseline and then again at 6 , 12 , and 18 months. The aim of the study was to determine the effects of the Green House model on residents' quality of life (via interviews) and quality of care (via MDS data). After controlling for baseline characteristics (such as age, gender, activities of daily living, date of admission) there was a statistically significant improvement in Green House residents' perception of their quality of life, compared with the control groups. ${ }^{30}$

\section{Facility-specific person-centered care}

Three studies (conducted in the UK, The Netherlands, and Australia) met the inclusion criteria for this review. ${ }^{26,32,33}$ Two of these facility-specific approaches ${ }^{26,32}$ evaluated the impact of person-centered interventions on organizational and workplace characteristics in addition to residents' wellbeing. One study ${ }^{32}$ confirmed that person-centered care positively impacted nurses' job satisfaction and work conditions, as well as improving their capacity to meet the individual needs of residents with dignity and respect. Furthermore, these person-centered approaches improved the continuity of residents' care because they were more likely to be assigned to the same nursing staff ${ }^{26}$ and also led to increased social interaction between residents. ${ }^{32}$

A large Australian study ${ }^{33}$ randomly assigned 289 residents across 15 aged-care facilities in Sydney, NSW, to receive person-centered care, dementia care mapping, or usual care. The facilities were selected because they adopted a task-focused, rather than a person-centered, approach to aged care and were similar in terms of management structures, staffing, standards, and size. Two staff from each facility received a two-day training session on how to promote person-centered care. The training emphasized the importance of engaging residents in meaningful social activities. The second intervention was dementia care mapping, which is an observational tool (developed at the University of Bradford, UK) designed to capture information about the experience of people with dementia in care settings. Staff and researchers using the tool can record information about the resident's level of activity, well-being, and psychosocial state. In the intervention groups, individual care plans were developed by nursing staff for residents. The control group received usual care, which was characterized by "custodial and physical taskoriented practices". Agitation was significantly lower with both person-centered and dementia care mapping than usual care. However, the incidence of falls was higher $(P=0.03)$ in person-centered care than in usual care.

\section{Discussion}

The shift towards a humanistic social model of aged care that embraces person-centered care as its core philosophy is part of a culture change movement influencing residential aged-care practices in several countries, including the US, UK, and The Netherlands. ${ }^{26,28,32}$ Rigorous evaluation of the impact of person-centered approaches on aged-care residents has not been a priority among operators of aged-care facilities, nor of researchers and gerontologists. Although in theory person-centered approaches to aged care should create the conditions for older people to participate in meaningful lives, and potentially improve their well-being, there is a paucity of studies designed to test this supposition.

To date, six quasi-experimental studies have evaluated the impact of person-centered interventions on aged-care residents and/or nursing staff, and their findings have been published in peer-reviewed journals. As Table 2 shows, most of these studies suffered design weaknesses that limit the inferences that can be made about these findings and the extent to which the results can be generalized. Furthermore, the studies utilized different data collection methods and instruments ${ }^{26,29,30,32}$ (data about validity indices were not provided), and ranged in duration from one to 3 years. ${ }^{28,32}$ Outcome measures (ie, dependent variables) included the impact of the Eden Alternative on residents' psychological status, ${ }^{28}$ functional status and infection rates, ${ }^{29}$ levels of depression and satisfaction with staffing care, ${ }^{31}$ the Green House model on residents' satisfaction, ${ }^{30}$ and facility-specific person-centered care approaches on the capacity of staff to deliver individualized care, ${ }^{32}$ their perception of their job characteristics, ${ }^{25,26}$ residents' views about quality of care, ${ }^{27}$ 
and levels of agitation in residents with dementia. ${ }^{33}$ One study failed to provide data on the sample size. ${ }^{31}$

Notwithstanding these variations and limitations in study designs, person-centered interventions in residential agedcare facilities were associated with psychosocial benefits to residents and staff. In one study, adoption and implementation of the Eden Alternative led to a significant reduction in residents' levels of boredom and helplessness, when assessed using the Geriatric Depression Scale. ${ }^{28}$ Another study of the Eden Alternative found a reduction in residents' level of depression according to the Geriatric Depression Scale and Cornell Depression Scale. ${ }^{31}$ Facility-specific approaches to person-centered care were associated with improvements in staff satisfaction levels and in their confidence and ability to provide high-quality individualized care..$^{26,32}$

Conflicting findings about the benefits to residents of person-centered care exist. Two studies ${ }^{29,33}$ found that a higher rate of falls occurred in residents receiving person-centered care interventions compared with residents receiving usual or traditional aged care. The higher rate of falls observed by Coleman et al, ${ }^{29}$ in the Eden Alternative facility might reflect the increased risk of accidents and injuries among ambulatory, independent residents compared with the frailer, more sedentary residents in the control group. Whereas both the Eden Alternative and traditional (control) aged-care facilities experienced staffing problems, the turnover of nursing staff was higher in the Eden Alternative facility, than in the control facility. Without information about the process used to induct, educate, and support staff to implement the principles in the Eden Alternative, the reason for the higher staff turnover is unclear.

In contrast with the study by Coleman et al, ${ }^{29}$ the higher rate of falls in the study by Chenoweth et al ${ }^{33}$ actually occurred in the residents with the highest care needs. One possible explanation for these findings is that the sites providing person-centered care were rated as being more hazardous than the other intervention sites using the "Therapeutic Environment Screening Survey for Nursing Homes". In other words, the facilities where person-centered care was adopted were viewed as being "less safe" when scored on the safety of the floor surfaces, ie, slipperiness, and the presence of handrails.

Person-centered interventions such as the Eden Alternative and the Green House model challenge traditional models of care and management because they are based on a collaborative, whole-of-facility management system. These models aim to empower the staff and place decision-making authority as close to the resident as possible. They "flatten" the nursing organization hierarchy by adopting a decentralized team method of care delivery that puts the resident at the center of the facility. ${ }^{34,35}$ The locus of decision-making is with the resident, which ensures that his/her wishes and desires are respected and valued. Nurturing relationships between frontline nurses and residents are of central importance in these culture change approaches.

Studies have shown that the successful implementation of culture change models requires good leadership and stable management; strong teamwork, efficient communication systems; and an investment in staff training and education about culture change. ${ }^{35,36}$ The Eden Alternative is based on ten principles, which provide a framework for person-centered, organizational culture change. The final principle emphasizes the importance of leadership in realizing effective, sustainable culture change. Resistance from senior leadership is the most frequently cited barrier to adopting culture change, followed by perceived cost and concern about compliance with regulatory requirements. ${ }^{12}$

As previously stated the majority of studies in this review were quasi-experimental designs. Quasi-experimental studies do not use randomization; consequently the results are less robust because they cannot eliminate the possibility of confounding bias. The aged-care setting makes the randomization of residents to an intervention or control group often impractical or unworkable. The control and intervention groups varied at baseline for age, ethnicity, and/or dependency status for the three studies. ${ }^{28-30}$ Selection bias is one of the main threats to the internal validity of quasi-experimental designs if the residents assigned to the intervention or control group vary in terms of their baseline demographic and health characteristics. Performance bias may arise if the researcher, participant or both, are aware of what treatment (or control) has been assigned. However, eliminating performance bias conflicts with the objectives of person-centered interventions, which promote a shared responsibility among staff to develop meaningful relationships with residents so they can understand their physical and psychosocial needs. As a result, it is neither possible nor appropriate to blind residents (participants) or staff to treatment allocation. Similarly, when environmental enhancement occurs simultaneously with other person-centered interventions (eg, the Eden Alternative), residents and staff cannot be blinded to the intervention.

The findings from this review have important implications for future research. In particular, future efforts to evaluate person-centered interventions critically are contingent upon researchers providing detailed information about the scope and nature of the intervention adopted. More specifically, in 
relation to the Eden Alternative, researchers need to identify and report the extent to which the ten principles have been implemented. Superficial culture change interventions, such as environmental enhancement only (principle 2), fail to address the need to enhance residents' social capacity through close and continuing relationships with staff, other residents and children (principle 2) and their engagement in meaningful activities (principles 3-6) that improve their quality of life. Some agreement on the outcome measures, evaluated using validated instruments that are shown to be sensitive to the overall intervention, or specific intervention components, would permit a comparison of study findings.

In light of the issue concerning increased incidence of falls in person-centered care facilities, future studies should aim to conduct an environmental safety audit on personcentered facilities and to have control and intervention groups that are comparable in terms of comorbid diseases, cognition, (instrumental) activity of daily living scores, and previous risk of falls. Alternatively, the scope of person-centered care should be expanded to include resident safety in an explicit way. Perhaps the features of person-centered care need to be framed within Maslow's hierarchy of needs to ensure that the basic building blocks of physiological needs and resident safety are met before the higher level functions are explored. Finally, information about staffing profiles, such as staff turnover and stability of management, would provide insight into the organizational culture and capacity to adopt and implement a sustainable person-centered intervention.

In summary, it appears that some person-centered interventions benefit residents and staff. However, person-centered care is a complex intervention, which presents several challenges for its evaluation and transferability. In order to be able to compare the benefits of the different approaches, there is a need for further exploration of the features of person-centered care and the use of a uniform language to describe the activities that underscore these interventions. These interventions then need to be made explicit so that the context can be understood and compared across studies. In terms of evaluation, there is a greater need for adoption of a standardized study design and development and/or adoption of instruments that will capture meaningfully the impacts of the intervention on the range of stakeholders. As these studies have shown, it is important to embed a study design that captures the positive and negative unintended consequences of the intervention.

\section{Conclusion}

The movement away from an institutional model of care to one that accepts person-centered care as the guiding standard of practice is part of a culture change that is impacting the provision of aged-care services around the world. Forming accurate conclusions about the impact of person-centered interventions on residents and staff is hampered by the heterogeneity of the interventions and significant methodological differences between studies. Person-centered interventions are associated with positive influences on staff outcomes (satisfaction and capacity to provide individualized care); improvement in the psychological status of residents (lower rates of boredom and feelings of helplessness); and reduced levels of agitation in residents with dementia. It appears that some person-centered interventions might be associated with an increased risk of falls in aged-care residents.

\section{Disclosure}

The authors have no conflicts of interest to report.

\section{References}

1. Rosher RB, Robinson S. Impact of the Eden Alternative on family satisfaction. J Am Med Dir Assoc. 2005;6:189-193.

2. McCallum A. Australian Population Association. What will we do with the baby boomers? Population globalisation: Australia in the 21st century. Available from: http://www.apa.org.au/upload/2000-2001B_ McCallum.pdf. Accessed November 19, 2012.

3. Benevolent Society. Baby boomers want alternatives to a future in nursing homes. Available from: http://www.bensoc.org.au/director/ newsandevents/mediareleases.cfm?item_id=FDED3B07DDFF3EB3 67B514F453C86210. Accessed November 19, 2012.

4. Kane RA. Definition, measurement, and correlates of quality of life in nursing homes: toward a reasonable practice, research, and policy agenda. Gerontologist. 2003;43:28-36.

5. Rogers C. A Way of Being. Boston, MA: Houghton Mifflin Company; 1980.

6. Crandall LG, White DL, Schuldheis S, Talerico KA. Initiating personcentered care practices in long-term care facilities. J Gerontol Nurs. 2007;33:47-56.

7. Thomas W, Johansson C. Elderhood in Eden. Top Geriatr Rehabil. 2003;19:282-290.

8. Stone R, Reinhard S, Bowers B, et al. Evaluation of the Wellspring Model for Improving Nursing Home Quality. Ithaca, NY: Institute for the Future of Aging Studies, Cornell University; 2002.

9. Tesh AS, McNutt K, Courts NF, Barba BE. Characteristics of nursing homes adopting environmental transformations. $J$ Gerontol Nurs. 2002;28:28-34.

10. Doty MM, Koren M, Sturla EL. Culture change in nursing homes: how far have we come? Findings from the Commonwealth Fund 2007 National Survey of Nursing Homes: The Commonwealth Fund; 2008. Available from: http://www.commonwealthfund.org/Publications/ Fund-Reports/2008/May/Culture-Change-in-Nursing-Homes--HowFar-Have-We-Come--Findings-From-The-Commonwealth-Fund-2007Nati.aspx. Accessed November 19, 2012.

11. Bond G, Fiedler F. A comparison of leadership vs renovation in changing staff values. Nurs Econ. 1999;17:37-43.

12. Miller SC, Miller EA, Jung HY, Sterns S, Clark M, Mor V. Nursing home organizational change: the "culture change" movement as viewed by long-term care specialists. Med Care Res Rev. 2010;67: 65S-81S.

13. Deutschman MT. An ethnographic study of nursing home culture to define organizational realities of culture change. J Health Hum Serv Adm. 2005;28:246-281. 
14. White DLP, Newton-Curtis L, Lyons KSP. Development and initial testing of a measure of person-directed care. Gerontologist. 2008;48: $114-123$.

15. MacKenzie S. Implementing the Eden Alternative in Australia. J Soc Work Long-Term Care. 2003;2:325-338.

16. Caspar S, O'Rourke N, Gutman GM. The differential influence of culture change models on long-term care staff empowerment and provision of individualized care. Can J Aging. 2009;28:165-175.

17. Brown Wilson C. Developing community in care homes through a relationship-centred approach. Health Soc Care Community. 2009;17: 177-186.

18. Hinman MR, Heyl DM. Influence of the Eden Alternative on the functional status of nursing home residents. Phys Occup Ther Geriatr. 2002;20:1-20.

19. Berkhout AJM, Boumans NPG, Mur I, Nijhuis FJN. Conditions for successfully implementing resident-oriented care in nursing homes. Scand J Caring Sci. 2009;23:298-308.

20. Chapman SA, Keating N, Eales J. Client-centred, community-based care for frail seniors. Health Soc Care Community. 2003;11:253-261.

21. Ransom S. The Eden Alternative: creating human habits in Texas nursing homes. Texas J Aging. 1998;1:8-13.

22. Drew JC, Brooke V. Changing a legacy: the Eden Alternative nursing home. Ann Long-Term Care. 1999;7:115-121.

23. Renick O, Ransom S. The search for Eden: an alternative path for nursing homes. Long-Term Care Interface. 2001;2:45-48.

24. Joanna Briggs Institute. Joanna Briggs Insitute Reviewers' Manual: 2011 Edition. Adelaide, South Australia: Joanna Briggs Institute, The University of Adelaide; 2011.

25. Berkhout AJM, Boumans NPG, Van Breukelen GPJ, Abu-Saad HH, Nijhuis JN. Resident-oriented care in nursing homes: effects on nurses. J Adv Nurs. 2004;45:621-632.
26. Berkhout AJM, Boumans NPG, Nijhuis FJN, Van Breukelen GPJ, Abu-Saad HH. Effects of resident-oriented care on job characteristics of nursing caregivers. Work Stress. 2003;17:337-353.

27. Boumans N, Berkhout A, Landeweerd A. Effects of resident-oriented care on quality of care, wellbeing and satisfaction with care. Scand J Caring Sci. 2005;19:240-250.

28. Bergman-Evans B. Beyond the basics: effects of the Eden Alternative model on quality of life issues. J Gerontol Nurs. 2004;30:27-34.

29. Coleman MT, Looney S, O’Brien J, Ziegler C, Pastorino CA, Turner C. The Eden Alternative: findings after 1 year of implementation. J Gerontol A Biol Sci Med Sci. 2002;57:M422-M427.

30. Kane RA, Lum TY, Cutler LJ, Degenholtz HB, Yu T. Resident outcomes in small-house nursing homes: a longitudinal evaluation of the initial Green House program. J Am Geriatr Soc. 2007;55:832-839.

31. Robinson SB, Rosher RB. Tangling with the barriers to culture change: creating a resident-centered nursing home environment. J Gerontol Nurs. 2006;32:19-27.

32. Ashburner C, Meyer J, Johnson B, Smith C. Using action research to address loss of personhood in a continuing care setting. Illn Crises Loss. 2004; 12:23-37.

33. Chenoweth L, King MT, Jeon YH, et al. Caring for Aged Dementia Care Resident Study (CADRES) of person-centred care, dementia-care mapping, and usual care in dementia: a cluster-randomised trial. Lancet Neurol. 2009;8:317-325.

34. Keane B. Building the new culture of ageing - one leader at a time. Nurs Homes. 2004;53:44-46.

35. Barba BE, Tesh AS, Courts NF. Promoting thriving in nursing homes: the Eden Alternative. J Gerontol Nurs. 2002;28:7-13.

36. Steiner JL, Eppelheimer C, De Vries M. Successful Edenization through education. Nurs Homes. 2004;53:46-49.
Clinical Interventions in Aging

\section{Publish your work in this journal}

Clinical Interventions in Aging is an international, peer-reviewed journal focusing on evidence-based reports on the value or lack thereof of treatments intended to prevent or delay the onset of maladaptive correlates of aging in human beings. This journal is indexed on PubMed Central, MedLine, the American Chemical Society's 'Chemical Abstracts

\section{Dovepress}

Service' (CAS), Scopus and the Elsevier Bibliographic databases. The manuscript management system is completely online and includes a very quick and fair peer-review system, which is all easy to use. Visit http://www.dovepress.com/testimonials.php to read real quotes from published authors. 